\title{
Modelos matemáticos em ecologia das populações: uma abordagem para estudantes de graduação
}

\author{
Mathematical models in population ecology: an approach to \\ undergraduate students
}

\author{
1 Mauro Sérgio Cruz Souza Lima \\ 1 Edivaldo Leal Queiroz \\ 2 Jonas Pederassi jonaspederassi@yahoo.com.br \\ 3 Carlos Alberto dos Santos Souza
}

\author{
Universidade Federal do Piauí, UFPI. \\ Museu Nacional, UFRJ. \\ 3 Universidade Federal de Juiz de Fora, UFJF.
}

\section{Resumo}

Os modelos matemáticos em ecologia podem ser de difícil compreensão ao graduando e os softwares destinados ao tratamento dos dados nem sempre auxiliam nesse entendimento. Propomos aqui abordar os modelos de Crescimento Exponencial, Crescimento Logístico, Predador-presa e Crescimento Estruturado em planilhas Excel $\circledast$, de modo que o aluno possa visualizar, de forma menos abstrata, seu funcionamento e aplicação. Para tanto, exemplificamos com resoluções de exercícios relacionados a cada modelo.

\section{Palavras-chave}

Modelagem; Ecologia; Thomas Malthus; Lotka-Volterra; Verhulst.

\section{Abstract}

The mathematic models adopted in ecological studies are often complex and might be of great challenge for the understanding of undergraduate students. Additionally, the software used in ecologic modeling are, in general, non obvious and of difficult comprehension. We propose an alternative MS Excel ${ }^{\circledR}$ approach to perform studies on Exponential Growth, Logistic Growth, Structured Growth and Predator-Prey models aiming to provide concrete understanding on the applications and functionalities of these models. We exemplify the use of ecologic modeling by providing solved exercises related to each one of the previously mentioned topics.

\section{Key words}

Modelling, Ecology, Thomas Malthus, Lotka-Volterra, Verhulst

\section{Como você deve citar?}

PEDERASSI, Jonas et al. Modelos matemáticos em ecologia das populações: uma abordagem para estudantes de graduação. Cadernos UniFOA, Volta Redonda, n. 32, p. 123-138, dez. 2016. 


\section{INTRODUÇÃO}

O graduando, ao se deparar com os modelos matemáticos sobre populações ecológicas, os considera teóricos, e a maioria, quando exerce alguma atividade prática de cálculo, utiliza softwares com entrada de dados, que torna difícil a compreensão do real motivo das variáveis que compõem os modelos. É necessário que o graduando entenda inicialmente o que vem a ser modelo e população, isto é, o modelo matemático que deriva do latim modellum, diminutivo de módus, que significa "medida que não se deve ultrapassar, baseados em experimentos e leis físicas" (HOUAISS, 2009). Modelos matemáticos buscam predizer e descrever o comportamento de um sistema (ALMEIDA \& BRITO, 2005), sendo um instrumento que valida hipóteses, a fim de desenvolver estratégias de intervenção (BUENDÍA, 2006). Por outro lado, as populações biológicas abrigam um grupo de atributos que podem ser quantitativamente definidos em condições experimentais ou de campo e consistem em qualquer grupo de organismos em determinado tempo e espaço específico (MARGALEF, 1982; ODUM, 1988; PINTO-COELHO, 2000; DAJOZ, 2005).

O presente estudo visa elucidar a aplicação dos modelos de Ecologia das Populações, com uso do software Microsoft Excel ${ }^{\circledR}$, de forma a colaborar com disciplinas introdutórias em Ecologia.

\section{MATERIAL E MÉTODOS}

Dentre os modelos matemáticos exemplificados neste trabalho, estabelecemos uma cronologia temporal para o entendimento sequencial, a fim de demonstrar como estudos anteriores influenciaram estudos posteriores. Sendo assim, foram abordados o crescimento exponencial Thomas Malthus 1798, o crescimento logístico Pierre-François Verhulst 1838, o modelo predador-presa de Alfred J. Lotka e Vito Volterra, na década de 1920 e o crescimento estruturado de Edward S. Deevey, na década de 1940 (DEEVEY \& DEEVEY, 1945; DEEVEY, 1947). Os modelos matemáticos e suas respectivas fórmulas foram construídos em Excel®. Exemplos para aplicação foram obtidos consultando-se a literatura.

\section{Modelos}

\section{Crescimento exponencial}

$N_{t}=N_{0} e^{t}$, assim temos:

$N=$ número de indivíduos em certa área geográfica no instante $t$

$r=$ constante que representa a taxa relativa de crescimento

\section{- Construção do modelo em planilha (e.g. Excel $\left.{ }^{\circledR}\right)$}

Tabela 1 - Modelo de planilha para crescimento exponencial

\begin{tabular}{ccc}
\hline & A & B \\
\hline 1 & $\mathrm{~N}_{0}$ & $\mathrm{t}_{\text {duplicação }}$ \\
\hline 2 & $\mathrm{~N}_{1}$ & $\mathrm{r}^{\mathrm{t}}$ \\
\hline 3 & $\mathrm{~N}_{2}$ & \\
\hline
\end{tabular}




\section{- Elementos do modelo em planilha (e.g. Excel®)}

$N_{o}$ - população inicial

$N_{1}$ - população no momento $1\left(N_{t}\right)$

$t_{\text {duplicąăo }}$ - cálculo do crescimento exponencial

$N_{t}=N_{0} e^{\text {tr }}-$ cálculo da constante da taxa de crescimento relativo

$\frac{\ln N_{t}}{N_{0}}=\ln \left(e^{r t}\right) \rightarrow \ln \frac{N_{t}}{N_{0}}=r^{t}$

\section{- Exemplos para aplicação:}

a. Crescimento exponencial de uma manada de 50 vacas, com $r=0,365$ vacas/(vaca * ano) (GOTELLI, 2007);

a. Durante cinco dias consecutivos, foi medido o tamanho de uma população de planárias em crescimento, como 100, 158, 315 e 794 indivíduos. Construa o gráfico do logaritmo (base $e$ ) do tamanho da população e estime o valor de $r$ (GOTELLI, 2007).

Crescimento logístico

$\frac{d N_{1}}{d t}=\frac{r N_{1}\left(K-N_{1}-\alpha N_{2}\right)}{N_{1}} \quad \frac{d N_{2}}{d t}=\frac{r N_{2}\left(K-N_{2}-\beta N_{1}\right)}{N_{2}}$, assim temos:

$\alpha$ (alfa) - coeficiente de competição ou fator de conversão de indivíduos da espécie 2 em unidades equivalentes da espécie 1

$\beta$ (beta) - coeficiente de competição ou fator de conversão de indivíduos da espécie 1 em unidades equivalentes da espécie 2

$N_{1}$ e $N_{2}$ - Tamanho das populações 1 e 2

$t$ - tempo

$r_{1}$ e $r_{2}$ - taxa intrínseca de crescimento per capta das espécies 1 e 2

$K_{1}$ e $K_{2}$-capacidade de suporte do meio ou densidade assintótica prevista pelo modelo logístico para as espécies 1 e 2 . 


\section{- Construção do modelo em planilha (e.g. Excel®)}

Tabela 2 - Modelo de planilha para crescimento logístico

\begin{tabular}{|c|c|c|c|c|c|c|}
\hline & A & B & $C$ & $\mathrm{D}$ & $E$ & $\mathrm{~F}$ \\
\hline 1 & & \multirow{11}{*}{ Valores } & $\mathrm{dN}_{1} / \mathrm{dt}$ & $\mathrm{dN}_{2} / \mathrm{dt}$ & $\mathrm{N}_{1}$ & $\mathrm{~N}_{2}$ \\
\hline 2 & $\mathrm{~N}_{1}$ & & \multirow{10}{*}{ Equação $_{1}$} & \multirow{10}{*}{ Equação $_{2}$} & \multirow{10}{*}{ População $_{1}$} & \multirow{10}{*}{ População $_{2}$} \\
\hline 3 & $\mathrm{r}_{1}$ & & & & & \\
\hline 4 & $\mathrm{k}_{1}$ & & & & & \\
\hline 5 & $a$ & & & & & \\
\hline 6 & $\mathrm{~N}_{2}$ & & & & & \\
\hline 7 & $r_{2}$ & & & & & \\
\hline 8 & $\mathrm{k}_{2}$ & & & & & \\
\hline 9 & $\beta$ & & & & & \\
\hline 10 & & & & & & \\
\hline 11 & & & & & & \\
\hline
\end{tabular}

- Elementos do modelo em planilha (e.g. Excel $\left.{ }^{\circledR}\right)$

$$
\frac{d N_{1}}{d t}=\frac{r N_{1}\left(K-N_{1}-\alpha N_{2}\right)}{N_{1}}
$$

- Procedimentos de cálculo em planilha (e.g. Excel $\left.{ }^{\circledR}\right)$

Equação $o_{1}=(\$ B \$ 3 * \$ B \$ 2((\$ B \$ 4-C 2-(D 2 * \$ B \$ 5)) /(\$ B \$ 2)$

Equação ${ }_{2}=(\$ B \$ 7 * \$ B \$ 6((\$ B \$ 8-D 2-(C 2 * \$ B \$ 9)) /(\$ B \$ 6)$

$d N_{1} / d t=(E 2+C 2)$

$d N_{2} / d t=(F 2+D 2)$

- Exemplos para aplicação:

a. Para a construção do modelo em questão, utilizamos a propositura de Delmas (2004) que compara a evolução da população belga em Verhulst (1845) e Verhulst (1847) com $\left(p(t)^{\prime}\right.$, assíntota $\left.A^{\prime}=6.6\right)$ e na segunda memória $\left(p(t)\right.$ ", assíntota $\left.A^{\prime \prime}=9.44\right)$;

b. Estudando uma população de tartarugas com denso-dependência, foram estabelecidas as seguintes relações da taxa de natalidade $b$ ' e taxa de mortalidade $d^{\prime}$ ', com o tamanho da população $(N)$ (GOTELLI, 2007), assim:

$b^{\prime}=0,10+0,03 N-0,0005 N^{2}$

$d^{\prime}=0,20+0,01 N$ 
Modelo predador-presa

$\frac{d P}{d t}=\beta V P-q P \quad \frac{d V}{d t}=r V-\alpha V P$, assim temos:

$P$ - população de predadores

$V$ - população de presas

$r$ - taxa intrínseca de crescimento

$q$ - taxa de mortalidade per capita

$\alpha$ - taxa de mortalidade da presa

$\beta$ - eficiência de conversão

\section{- Construção do modelo em planilha (e.g. Excel $\left.{ }^{\circledR}\right)$}

Tabela 3 - Modelo de planilha para modelo predador-presa

\begin{tabular}{|c|c|c|c|c|c|c|c|c|c|c|c|}
\hline & $\mathbf{A}$ & $\mathbf{B}$ & $\mathbf{C}$ & $\mathbf{D}$ & $\mathbf{E}$ & $\mathbf{F}$ & $\mathbf{G}$ & $\mathbf{H}$ & $\mathbf{I}$ & $\mathbf{J}$ & $\mathbf{K}$ \\
\hline $\mathbf{1}$ & $t$ & Presa $V(t)$ & $\begin{array}{c}\text { Predador } \\
r P(t)\end{array}$ & $V^{\prime}(t)$ & $P^{\prime}(t)$ & $V(t+0.5)$ & $P(t+0.5)$ & $V^{\prime}(t+0.5)$ & $P^{\prime}(t+0.5)$ & $r$ & $*$ \\
\hline $\mathbf{2}$ & 0 & & & & & & & & & $\alpha$ & $*$ \\
\hline $\mathbf{3}$ & 1 & & & & & & & & & $\beta$ & $*$ \\
\hline $\mathbf{4}$ & & & & & & & & & & $q$ & $*$ \\
\hline
\end{tabular}

\section{- Elementos do modelo em planilha (e.g. Excel $\left.{ }^{\circledR}\right)$}

* Parâmetros: cada população descreve um ciclo com amplitudes determinadas pelos tamanhos populacionais iniciais e com período de aproximadamente $\frac{2 \pi}{\sqrt{r q}}$, Gotelli (2007).

\section{- Procedimentos de cálculo em planilha (e.g. Excel $\left.{ }^{\circledR}\right)$}

$V(t)=(B 2+H 2)$

$P(t)=(C 2+I 2)$

$V^{\prime}(t)=((\$ K \$ 1 * B 2)-(\$ K \$ 2 * B 2 * C 2))$

$P^{\prime}(t)=((\$ K \$ 3 * B 2 * C 2)-(\$ K \$ 4 * C 2))$

$V(t+0.5)=(B 2+\$ K \$ 4 * D 2)$

$P(t+0.5)=(C 2+\$ K \$ 4 * E 2)$ 
$V^{\prime}(t+0.5)=(\$ K \$ 1 * G 2-\$ K \$ 2 * G 2 * H 2)$

$P^{\prime}(t+0.5)=(\$ K \$ 3 * F 2 * G 2-\$ K \$ 4 * G 2)$

- Exemplos para aplicação:

a. Com base no artigo de Elton e Nicholson (1942), caso clássico em que utilizaram o modelo Lotka-Volterra e demonstraram que as populações de linces e lebres oscilam periodicamente, considere: Predador $=15 ;$ Presa $=50 ; r=0.1 ; \alpha=0.01 ; \beta=0.001 ; q=0.05$.

Crescimento estruturado

$T_{x}=E_{x}+E_{x+1}+E_{x+2}+\ldots+E_{w}$

$T_{x}$-é o número total de indivíduos de idade $x$ além da idade $x$, em que:

$X$ - intervalo de idade em unidades de tempo

$L_{x}-$ número de sobreviventes no começo da idade $x$

$d_{x}$ - número de indivíduos mortos durante o intervalo etário $x$

Ex - estrutura etária, onde:

$E_{x}=\frac{L_{x}+L_{x+1}}{2}$

$e x$ - é a esperança de vida para indivíduos de idade $x$, sendo encontrado por:

$e x=\frac{T_{x}}{L_{x}}$

1000qx- razão de mortalidade por intervalo de idade, onde:

$1000 q_{x}=1000 \frac{d_{x}}{L_{x}}$ 


\section{- Construção do modelo em planilha (e.g. Excel $\left.{ }^{\circledR}\right)$}

Tabela 4 - Modelo de planilha para crescimento estruturado

\begin{tabular}{|c|c|c|c|c|c|c|c|}
\hline & A & B & $\mathrm{C}$ & $\mathrm{D}$ & $\mathrm{E}$ & $\mathrm{F}$ & G \\
\hline 1 & $x$ & $L_{x}$ & $d_{x}$ & $E_{x}$ & $T_{x}$ & $e_{x}$ & $1000 q_{x}$ \\
\hline 2 & 1 & & & & & & \\
\hline 3 & 2 & & & & & & \\
\hline 4 & 3 & & & & & & \\
\hline 5 & 4 & & & & & & \\
\hline 6 & 5 & & & & & & \\
\hline 7 & 6 & & & & & & \\
\hline 8 & 7 & & & & & & \\
\hline 9 & 8 & & & & & & \\
\hline
\end{tabular}

- Elementos do modelo e procedimentos de cálculo em planilha (e.g. Excel®)

$X$ - intervalo de 1 a 8

$L x$ - número de sobreviventes no começo da idade $x$

$B 2=\operatorname{SOMA}(C 2: C 9) \rightarrow B 3=(B 2-C 2)$

$d x$ - número de indivíduos mortos durante o intervalo etário $x$ (valores iniciais)

$=(B 2+B 3 / 2)$

$E_{x}=\frac{L_{x}+L_{x+1}}{2}$

$T_{x}=E_{x}+E_{x+1}+E_{x+2}+\ldots+E_{w}$

$=S O M A(D 2: D \$ 9)$

$e_{x}=\frac{T_{x}}{L_{x}}$

$=(E 2 / B 2)$

$1000 q_{x}=1000 \frac{d_{x}}{L_{x}}$

$=(C 2 / B 2) * 1000$

\section{- Exemplos para aplicação:}

a. Tabela de vida proposta por Southwood (1971). 


\section{RESULTADOS E DISCUSSÃO}

\subsection{Crescimento exponencial}

No exercício de aplicação " $a$ " do crescimento exponencial Malthus 1798, é possível observar pelos resultados que quanto maior o $r$ menor é o tempo de duplicação (Figura 1). Populações consideradas pequenas, em virtude de crescerem exponencialmente, segundo o modelo, poderão atingir valores que ultrapassarão a própria condição ambiental (GOTELLI, 2007). Assim, em 150 anos, o crescimento exponencial da população de vacas corresponderá a $3 \times 10^{25}$ (Figura 1). Considerando que o peso de uma novilha madura sexualmente seja $300 \mathrm{~kg}$ (CANELLAS et al., 2010), aos 150 anos de crescimento exponencial, teríamos um valor superior ao peso do planeta Terra (GOTELLI, 2007).

Figura 1 - Planilha construída em Microsoft Excel®. Resultados referentes ao exercício de aplicação "a" do modelo de crescimento exponencial proposto em Gotelli (2007). Procedimentos de cálculo em planilha Excel ${ }^{\circledR}$ (fórmulas): $C 2=B 3 / B 2 ; C 3=\ln (C 2) ; B 4=B \$ 2 * E X P(C \$ 3 * A 4)$ * *Valores arredondados de Gotelli (2007).

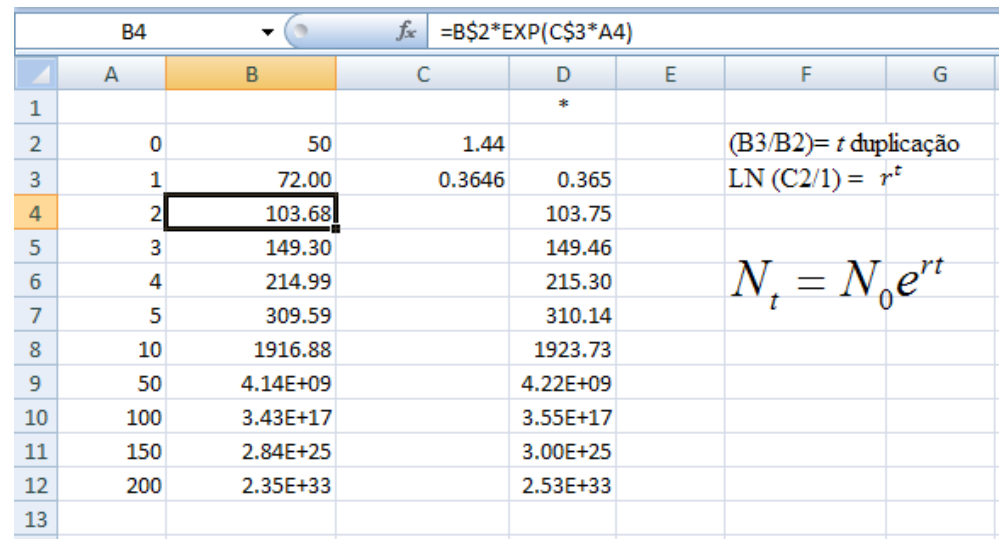

Fonte: dos autores.

No exercício de aplicação "b" sobre o crescimento exponencial de populações de planárias, a planilha em Excel® modelará um gráfico com uma reta, caracterizando o crescimento sem limitação de recursos (Figura 2). 0 valor de $r$ pode ser determinado medindo-se a inclinação da reta, sendo o valor correspondente $(r=0,6906)$ referente à taxa de crescimento relativo de planárias (Figura 2).

Figura 2 - Representação gráfica em Excel ${ }^{\circledR}$ dos valores da população de planárias logaritmizados a partir do exercício de aplicação "b" do modelo de crescimento exponencial proposto por Gotelli (2007).

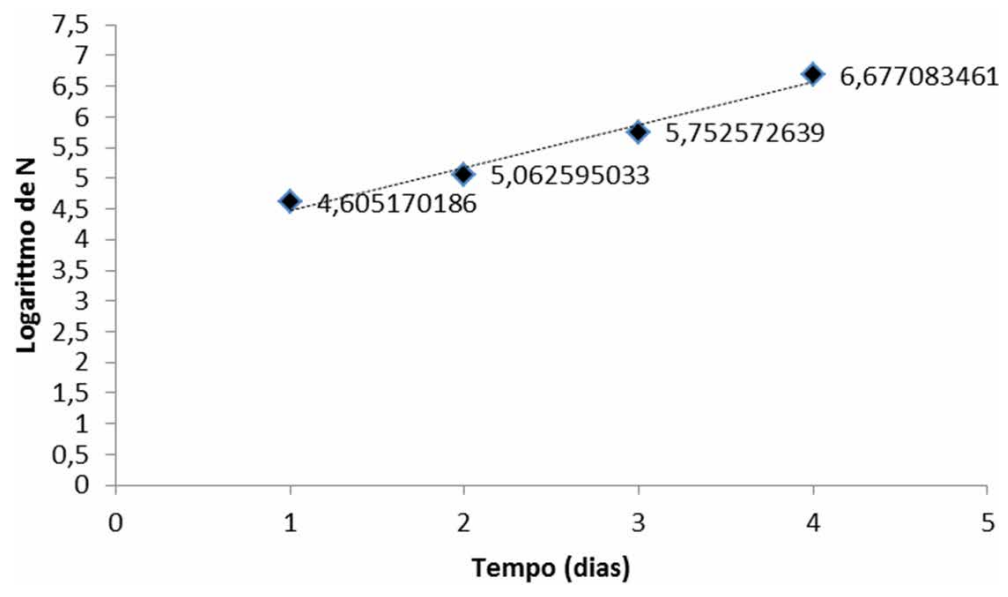

Fonte: dos autores. 
Ao avaliarmos o modelo de crescimento exponencial, fica evidenciado que, conforme seus postulados, "a capacidade de reprodução da população é superior à capacidade da terra para produzir meios de subsistência para o homem", entretanto acreditava-se que o controle da população humana ocorreria em observância aos fatos vivenciados na Grã-Bretanha, em plena Revolução Industrial. Dessa forma, o equilíbrio ocorreria naturalmente com o aumento da pobreza, abundância de trabalhadores, queda do valor da hora de trabalho, fazendo com que os pobres oscilassem em maior e menor número de descendentes (SZMRECSÁNYI, 1982).

O modelo de crescimento exponencial foi o primeiro modelo matemático a ser utilizado em Ecologia (ANGELINI, 2000), no entanto, inevitavelmente, a oferta de alimentos seria menor que a população. Porém, sabemos que as populações não crescem indefinidamente, pois os fatores bióticos e abióticos integrados funcionam como limitadores (RICKLEFS, 2010). A população, portanto, apresenta um parâmetro de controle, denominado em Ecologia como capacidade suporte, desenvolvido por Verhulst (1838), cuja variável K é a assíntota da curva e representa o máximo de crescimento da população em relação à disponibilidade dos recursos (e.g alimento, abrigo) (ANGELINI, 2000).

\subsection{Crescimento logístico}

Na construção em planilha Excel® do crescimento logístico Verhulst 1838 proposto por Delmas (2004), obteremos o resultado, conforme a Figura 3.

Figura 3 - Planilha construída em Microsoft Excel®. Resultados referentes ao exercício de aplicação "a" do modelo de crescimento logístico, proposto por Delmas (2004).

\begin{tabular}{|c|c|c|c|c|c|c|c|c|c|}
\hline 4 & A & B & C & D & $E$ & $\mathrm{~F}$ & G & H & 1 \\
\hline 1 & & & $\mathrm{dt}$ & $\mathrm{dN} 1 / \mathrm{dt}$ & $\mathrm{dN} 2 / \mathrm{dt}$ & & & Mortalidade & Natalidade \\
\hline 2 & N1 & 34 & 0 & & & & 1 & 6 & 34 \\
\hline 3 & r1 & 0,03 & 1 & 17,718 & 35,92 & & 2 & 10,20927547 & 69,92 \\
\hline 4 & K1 & 600 & 2 & 17,48396 & 32,328 & & 3 & 27,69323721 & 102,248 \\
\hline 5 & $\alpha$ & 0,1 & 3 & 16,86246 & 29,0952 & & 4 & 44,55569609 & 131,3432 \\
\hline 6 & N2 & 6 & 4 & 16,2693 & 26,18568 & & 5 & 60,82499561 & 157,52888 \\
\hline 7 & $\mathrm{r} 2$ & 0,1 & 5 & 15,70266 & 23,56711 & & 6 & 76,5276591 & 181,095992 \\
\hline 8 & K2 & 400 & 6 & 15,16088 & 21,2104 & & 7 & 91,68854135 & 202,3063928 \\
\hline 9 & $\beta$ & 0,2 & 7 & 14,64242 & 19,08936 & & 8 & 106,3309659 & 221,3957535 \\
\hline 10 & & & 8 & 14,14588 & 17,18042 & & 9 & 120,4768497 & 238,5761782 \\
\hline 11 & & & 9 & 13,66997 & 15,46238 & & 10 & 134,1468157 & 254,0385604 \\
\hline 12 & & & 10 & 13,21348 & 13,91614 & & 11 & 147,3602955 & 267,9547043 \\
\hline 13 & & & 11 & 12,77533 & 12,52453 & & 12 & 160,1356225 & 280,4792339 \\
\hline 14 & & & 12 & 12,35449 & 11,27208 & & 13 & 172,4901162 & 291,7513105 \\
\hline 15 & & & 13 & 11,95004 & 10,14487 & & 14 & 184,4401587 & 301,8961794 \\
\hline 16 & & & 14 & 11,56111 & 9,130382 & & 15 & 196,0012654 & 311,0265615 \\
\hline 17 & & & 15 & 11,18688 & 8,217344 & & 16 & 207,1881478 & 319,2439054 \\
\hline
\end{tabular}

Fonte: dos autores.

Na planilha em Excel ${ }^{\circledR}$, o modelo gráfico gerado corresponderá às colunas $N_{1}$ e $N_{2}$, onde fica evidenciada a capacidade suporte e as assíntotas geradas, conforme prevê o modelo de crescimento logístico (Figura 4).

No exercício de aplicação "b" (crescimento logístico) que estudou a denso-dependência entre a taxa de natalidade de tartarugas para a população $\mathrm{N}$, em taxa de natalidade $b^{\prime}=0.10+0.03 \mathrm{~N}-0.0005 \mathrm{~N}^{2} \mathrm{e}$ taxa de mortalidade de d' $=0.20+0.01 \mathrm{~N}($ GOTELLI, 2007), devemos aplicar os procedimentos de cálculos (fórmulas) em planilha Excel®, obtendo a Figura 5. 
Figura 4 - Representação gráfica em Excel®, a partir do exercício de aplicação "a" do modelo de crescimento logístico, segundo proposta de Delmas (2004). Assíntotas geradas a partir da variável K, que corresponde à capacidade suporte do ambiente em relação às populações $N_{1}$ e $N_{2}$.

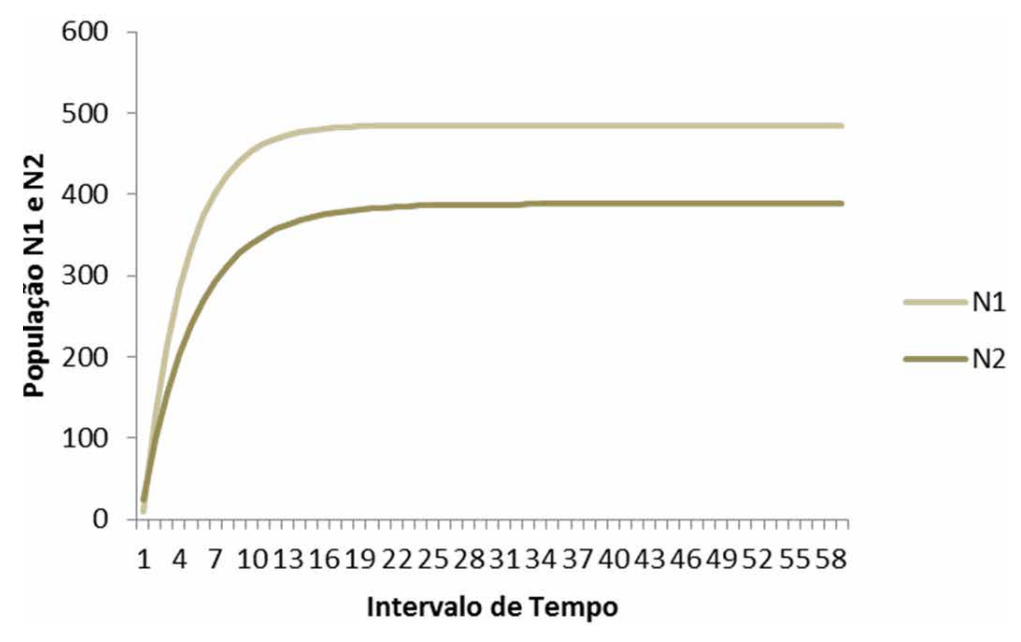

Fonte: dos autores.

Figura 5 - Planilha construída em Microsoft Excel®. Resultados referentes ao exercício de aplicação "b" do modelo de crescimento logístico, proposto em Gotelli (2007). Para natalidade, foi considerada a fórmula $b^{\prime}=0.10+0.03 \mathrm{~N}-0.0005 \mathrm{~N}^{2}$ e, para mortalidade, a fórmula $\mathrm{d}^{\prime}=0.20+0.01 \mathrm{~N}$, onde $\mathrm{N}$ corresponde à coluna $\mathrm{A}$.

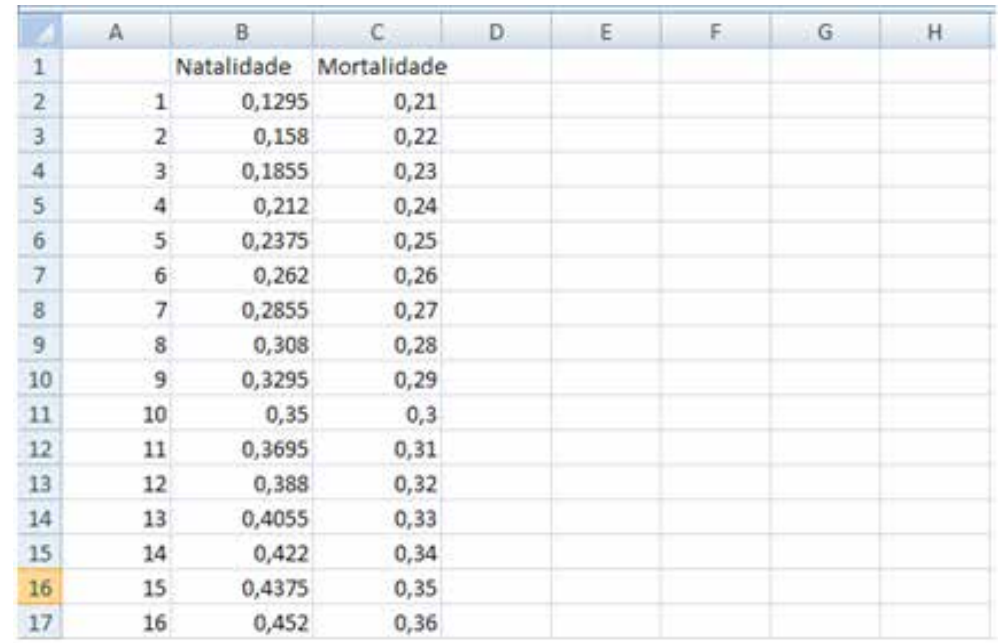

Fonte: dos autores.

A equação de mortalidade é linear, conforme prevê o modelo de crescimento logístico, enquanto a taxa de natalidade é quadrática, quando Gotelli (2007) inclui $\mathrm{N}^{2}$. Igualando as duas taxas propostas pelas equações $0.10+0.03 \mathrm{~N}-0.0005 \mathrm{~N}^{2}=0.20+0.01 \mathrm{~N}$, obteremos, respectivamente, $\mathrm{N}=6$ e N' $=34$. Dessa forma, ao construirmos o gráfico, ocorrerão duas interceptações entre as taxas de natalidade e mortalidade, sendo o primeiro ponto em 6 , correspondendo a 6 tartarugas. Assim, se a taxa de natalidade excede a taxa de mortalidade, a população crescerá até atingir o equilíbrio de 34 tartarugas (Figura 6). 
Figura 6 - Representação gráfica em Excel® a partir do exercício de aplicação "b" do modelo de crescimento logístico, segundo proposta de Gotelli (2007). Descrição das curvas de crescimento em relação às taxas de mortalidade, crescimento logístico simples e taxa de natalidade com crescimento quadrático.

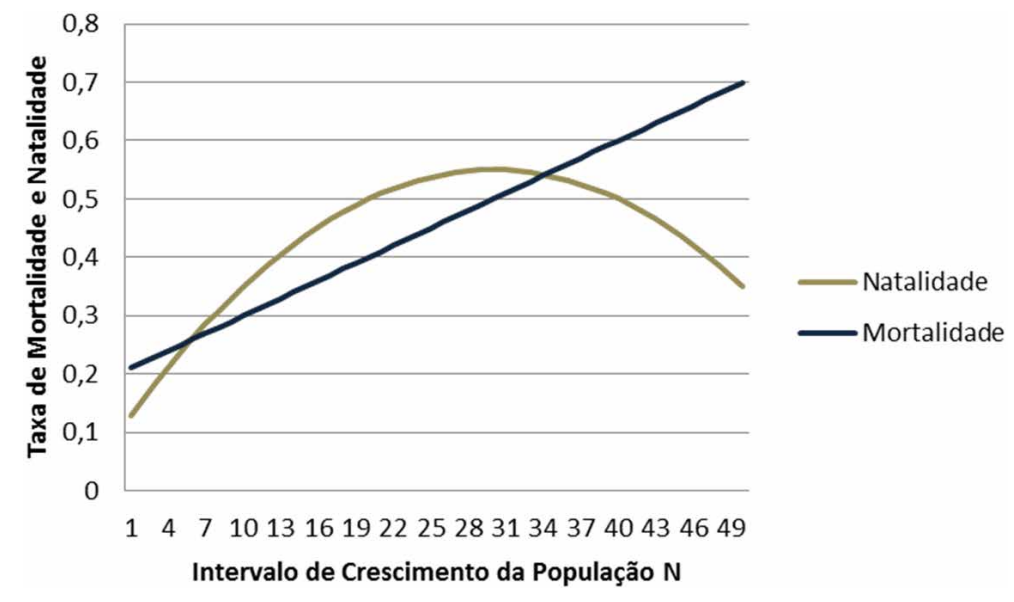

Fonte: dos autores.

O modelo de crescimento logístico estabeleceu que a população não crescesse indefinidamente, como o modelo de crescimento exponencial, mas obrigatoriamente apresenta um limite assintótico. Foi adotado, portanto, que a resistência ao crescimento é proporcional ao quadrado da velocidade de crescimento (DELMAS, 2004).

A equação que se contrapõe ao meio ou as opostas taxas de crescimento são elementos que favorecem o nascimento e a vida, contra os que favorecem as doenças e a morte. Dessa forma, podem ser representados matematicamente por $b-d$ (HIGLEY, 2002). Quando se considera a assíntota como a forma com que a população se apresentará estável, a partir de um limitador K, sabemos que a estabilidade das populações no ambiente não é estável, são de fato dinâmicas e oscilam entre os recursos disponíveis e o crescimento populacional. Por outro lado, no modelo de predador-presa que foi formulado independente (LOTKA, 1925; VOLTERRA, 1926 e 1931), descrevem-se mudanças oscilatórias entre duas populações que interagem.

\subsection{Modelo predador-presa}

Considerando os dados do exercício de aplicação "a" referente ao modelo predador-presa (Predador $=15$; Presa $=50 ; r=0.1 ; \alpha=0.01 ; \beta=0.001 ; q=0.05$ ), confeccionamos planilha Excel ${ }^{\circledR}$ (Figura 7). 
Figura 7 - Planilha construída em Microsoft Excel®. Resultados referentes ao exercício de aplicação "a" do modelo predador-presa, conforme proposta de Elton e Nicholson (1942). Evolução do N populacional predador/presa nas colunas $\mathrm{B}$ e $\mathrm{C}$ em denso-dependência, segundo os valores de $\mathrm{a}, \beta$ e $q$, desenvolvendo a fórmula nas colunas de $\mathrm{B}$ a I.

\begin{tabular}{|c|c|c|c|c|c|c|c|c|c|c|c|}
\hline & A & B & c & D & $\mathrm{E}$ & $\mathrm{F}$ & G & H & 1 & J & K \\
\hline 1 & Tempo & presa & Predador & & & & & & & & \\
\hline 2 & $t$ & $\mathrm{~V}(\mathrm{t})$ & $\mathrm{P}(\mathrm{t})$ & $V^{\prime}(t)$ & $P^{\prime}(t)$ & $V(t+0,5)$ & $P(t+0,5)$ & $v^{\prime}(t+0,5)$ & $P^{\prime}(t+0,5)$ & $r$ & 0,1 \\
\hline 3 & 0 & 50 & 15 & $-2,5$ & 0 & 49,875 & 15 & $-2,49375$ & $-0,00187$ & $\alpha$ & 0,01 \\
\hline 4 & 1 & 47,50625 & 14,998125 & $-2,37442$ & $-0,0374$ & 47,38753 & 14,99625 & $-2,3676$ & $-0,03918$ & $\beta$ & 0,001 \\
\hline 5 & 2 & 45,13865 & 14,95894772 & $-2,2384$ & $-0,07272$ & 45,02673 & 14,95531 & $-2,23121$ & $-0,07438$ & q & 0,05 \\
\hline 6 & 3 & 42,90743 & 14,88457089 & $-2,09584$ & $-0,10557$ & 42,80264 & 14,87929 & $-2,08847$ & $-0,10709$ & & \\
\hline 7 & 4 & 40,81897 & 14,77747928 & $-1,95012$ & $-0,13567$ & 40,72146 & 14,7707 & $-1,9427$ & $-0,13705$ & & \\
\hline 8 & 5 & 38,87627 & 14,64042882 & $-1,80403$ & $-0,16286$ & 38,78607 & 14,63229 & $-1,79668$ & $-0,16409$ & & \\
\hline 9 & 6 & 37,07959 & 14,47634337 & $-1,65981$ & $-0,18704$ & 36,9966 & 14,46699 & $-1,65263$ & $-0,18812$ & & \\
\hline 10 & 7 & 35,42695 & 14,28822328 & $-1,51919$ & $-0,20822$ & 35,35099 & 14,27781 & $-1,51225$ & $-0,20916$ & & \\
\hline 11 & 8 & 33,9147 & 14,07906753 & $-1,3834$ & $-0,22647$ & 33,84553 & 14,06774 & $-1,37675$ & $-0,22726$ & & \\
\hline 12 & 9 & 32,53796 & 13,85181064 & $-1,2533$ & $-0,24188$ & 32,47529 & 13,83972 & $-1,24696$ & $-0,24254$ & & \\
\hline 13 & 10 & 31,291 & 13,60927362 & $-1,12938$ & $-0,25462$ & 31,23453 & 13,59654 & $-1,12336$ & $-0,25515$ & & \\
\hline 14 & 11 & 30,16763 & 13,35412807 & $-1,01186$ & $-0,26484$ & 30,11704 & 13,34089 & $-1,00618$ & $-0,26526$ & & \\
\hline 15 & 12 & 29,16146 & 13,08887176 & $-0,90076$ & $-0,27275$ & 29,11642 & 13,07523 & $-0,8954$ & $-0,27306$ & & \\
\hline 16 & 13 & 28,26606 & 12,81581405 & $-0,79592$ & $-0,27854$ & 28,22626 & 12,80189 & $-0,79087$ & $-0,27874$ & & \\
\hline 17 & 14 & 27,47519 & 12,53706913 & $-0,69706$ & $-0,2824$ & 27,44034 & 12,52295 & $-0,69231$ & $-0,28251$ & & \\
\hline 18 & 15 & 26,78289 & 12,25455562 & $-0,60384$ & $-0,28452$ & 26,75269 & 12,24033 & $-0,59935$ & $-0,28455$ & & \\
\hline 19 & 16 & 26,18354 & 11,97000091 & $-0,51582$ & $-0,28508$ & 26,15775 & 11,95575 & $-0,51158$ & $-0,28505$ & & \\
\hline 20 & 17 & 25,67196 & 11,68494896 & $-0,43256$ & $-0,28427$ & 25,65033 & 11,67074 & $-0,42855$ & $-0,28418$ & & \\
\hline 21 & 18 & 25,24341 & 11,40077039 & $-0,3536$ & $-0,28224$ & 25,22573 & 11,38666 & $-0,34979$ & $-0,2821$ & & \\
\hline 22 & 19 & 24,89361 & 11,11867423 & $-0,27848$ & $-0,27915$ & 24,87969 & 11,10472 & $-0,27485$ & $-0,27895$ & & \\
\hline 23 & 20 & 24,61876 & 10,83972029 & $-0,20673$ & $-0,27513$ & 24,60843 & 10,82596 & $-0,20326$ & $-0,27489$ & & \\
\hline
\end{tabular}

Fonte: dos autores.

Na planilha em Excel ${ }^{\circledR}$, no modelo gráfico, obteremos suaves oscilações entre o predador e a predador (Figura 8).

Figura 8 - Representação gráfica em Excel ${ }^{\circledR}$ a partir do exercício de aplicação "a" do modelo de predador-presa, segundo Elton e Nicholson (1942). Gráfico gerado a partir das colunas B e C com a demonstração das curvas de oscilação denso-dependentes.

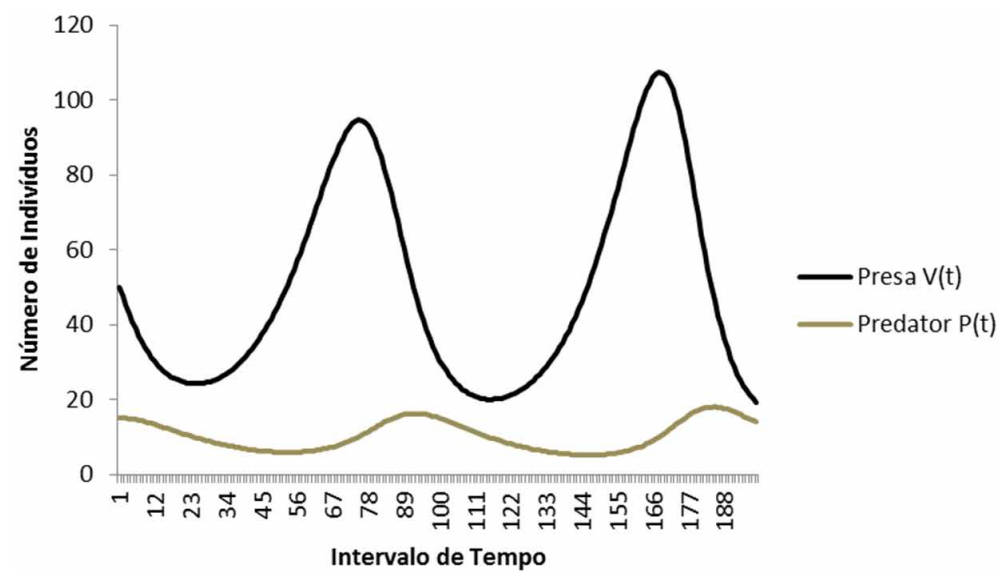

Fonte: dos autores.

Scott e Craine (1993) demonstraram, em seus estudos, que os trabalhos de Elton e Nicholson (1942) apresentavam falhas, ao analisarem as populações de linces e lebres, convalidando os diversos autores que apontaram falhas no modelo Lotka-Volterra (SHIH, 1997; CHAUVET et al., 2002; MOLTER \& RAFIKOV, 2004; SCHRUM, 2005). Os pesquisadores encontram, no modelo Lotka-Volterra, um sistema caótico, isto é, em sistemas dinâmicos complexos os resultados podem ser instáveis, no que se refere à condição temporal como função dos parâmetros das variáveis (SCOTT \& CRAINE, 1993). Dessa forma, 
buscam conduzir a trajetória das curvas para pontos desejados por meio de funções de otimidade na relação predador-presa (GILPIN, 1979; VANCE, 1998; MOLTER \& RAFIKOV, 2004).

O sistema predador-presa em denso-dependência pode ser perturbado por fatores externos, como: imigração de novos predadores e ações climáticas severas, logo, não há um modelo matemático que garanta resultados estáticos, pois o sistema presa predador é dinâmico e complexo, gerando o que se chama Sistema Caótico, isto é, dependendo dos dados, as trajetórias das curvas são instáveis e perdem o caráter de oscilações. Muito embora, o modelo Lotka-Voterra seja fonte de inspiração para muitos trabalhos que envolvam comunidades, segundo Gause (1934), sua validade só ocorre para experimentos laboratoriais, uma vez que as condições temporais estão mais sujeitas ao controle das variáveis (HALL, 1988).

\subsection{Crescimento estruturado}

O estudo de populações em laboratório, em especial a condição de longevidade e crescimento, se vale usualmente do modelo de crescimento estruturado proposto por Deevey, nas décadas de $1940 \mathrm{e}$ 1950. No exercício de aplicação "a" referente à tabela de vida de Southwood (1971) para o crescimento estruturado, temos a Figura 9.

Figura 9 - Planilha construída em Microsoft Excel®. Resultados referentes ao exercício de aplicação "a" do crescimento estruturado a partir da tabela de vida (SOUTHWOOD, 1971).

\begin{tabular}{|c|c|c|c|c|c|c|c|}
\hline 4 & A & B & C & D & E & $\mathrm{F}$ & G \\
\hline 1 & $x$ & Lx & $d x$ & Ex & Tx & ex & $1000 q X$ \\
\hline 2 & 1 & 1000 & 300 & 850 & 2180 & 2,18 & 300 \\
\hline 3 & 2 & 700 & 200 & 600 & 1330 & 1,90 & 285,71 \\
\hline 4 & 3 & 500 & 200 & 400 & 730 & 1,46 & 400 \\
\hline 5 & 4 & 300 & 200 & 200 & 330 & 1,10 & 666,67 \\
\hline 6 & 5 & 100 & 50 & 75 & 130 & 1,30 & 500 \\
\hline 7 & 6 & 50 & 30 & 35 & 55 & 1,10 & 600 \\
\hline 8 & 7 & 20 & 10 & 15 & 20 & 1,00 & 500 \\
\hline 9 & 8 & 10 & 10 & 5 & 5 & 0,50 & 1000 \\
\hline 10 & & & 1000 & & & & \\
\hline
\end{tabular}

Fonte: dos autores.

As taxas de natalidade e mortalidade estão intimamente relacionadas com a idade dos indivíduos, variando de espécie para espécie numa dada condição abiótica (ODUM, 1988; GOTELLI, 2007). As tabelas de vida demonstram a estrutura etária da população, bem como sua taxa de mortalidade para cada faixa etária (DAJOZ, 2005), sendo úteis no entendimento da estrutura etária das populações e suas características. A partir da coluna $\mathrm{L}_{\mathrm{x}^{\prime}}$ é possível traçar a curva de sobrevivência (Figura 10) em que, no exemplo, observa-se que a mortalidade se mantém aproximadamente constante para os estágios mais jovens. 
Figura 10 - Representação gráfica em Excel® a partir do exercício de aplicação "a" do crescimento estruturado. Curva de sobrevivência traçada a partir da tabela de vida, proposta por Southwood (1971).

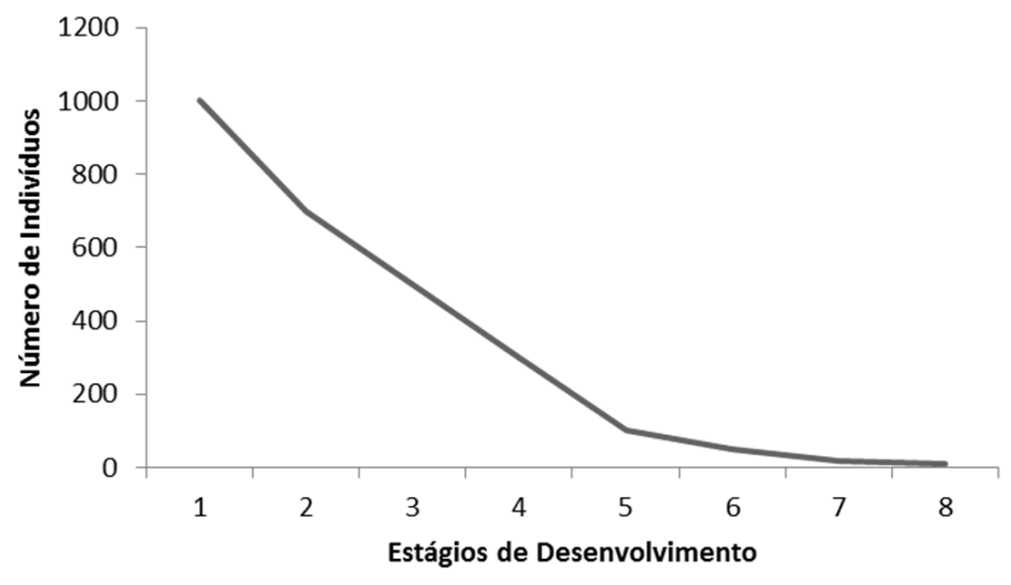

Fonte: dos autores.

Os mesmos dados, descritos em escala semilogarítmica, enquanto o intervalo de tempo expressar, em porcentagem, o tempo médio de vida, pode ser utilizado para a comparação de espécies com durações de vida distintas entre si (ODUM, 1988).

Os modelos devem servir para predizer, construir e gerar teorias gerais (LEGENDRE \& LEGENDRE 1998), tanto na Sinecologia quanto na Autoecologia, mas não se podem generalizar modelos e resultados, que, sem dúvida, são os principais aspectos de crítica pelos estudiosos (MANSSON \& MCGLADE, 1993; ANGELINI, 2000). Esperamos que com os resultados obtidos, os ensaios matemáticos modelados em Excel ${ }^{\circledR}$, segundo os postulados de modelagem ecológica, tenham garantido ao graduando entender as perspectivas de cada método, no que tange as suas próprias limitações e resultados e que a utilização desse software tenha servido para que as fórmulas de fato sejam compreendidas de maneira a permitir que se façam outras simulações. No entanto, caso exista interesse em simular sistemas de maior complexidade, sugerimos fazer uso dos softwares Ecopath ${ }^{\circledR}(2015)$ e Vensin ${ }^{\circledR}$ (2015). 


\section{REFERÊNCIAS}

ALMEIDA, L. M. W.; BRITO, D.S. Atividades de Modelagem Matemática: que sentido os alunos podem Ihe atribuir? Ciência \& Educação, v.11, n.3, p.483-498, 2005.

ANGELINI, R. Ecologia e modelagem matemática. Revista de Ciência Biológicas e Ambientais PUC-SP, v.2, n.2, p. 225-242, 2000.

BUENDÍA, G. Una socioepistemologia del aspecto periódico de las funciones. In: Revista Latinoamericana de Investigacion en Matematica Educativa, v.9, n.2, p. 227-251, 2006.

CANELLAS, L. C.; MARQUES, P. R.; BARCELLOS, J. O. J; LAMPERT, V.; NETO, J. B. Estimativa de custo de três sistemas alimentares para recria de novilhas acasaladas aos 18 meses de idade. Acta Scientiae Veterinariae, v.38, n.1, p.1-10, 2010.

CHAUVET, E.; PAULLET, J. E.; PREVITE, J. P.; WALLS, Z. A Lotka-Volterra three species food chain. Mathematics Magazine, v.75, n.4, p. 243-255, 2002.

DAJOZ, R. Princípios de ecologia. ed. 7. Artmed: Porto Alegre, Brasil. 2005. 519 pp.

DEEVEY, E. S. Life tables for natural populations of animals. The Quartely Review of Biology, v.22, p.283-314, 1947.

DEEVEY, E.S.; DEEVEY, G.B. A life table for the black widow. Transactions of the Connecticut Academy of Arts and Sciences, v.36, p.34-115, 1945.

DELMAS, B. Pierre-François Verhulst et la loi logistique de la population. Math Sci Hum / Mathematical Social Sciences, v.42, n.167, p.51-81, 2004.

ECOPATH, 2015. Ecopath with Ecosim: no fish is an island. Disponivel em: <http://www.ecopath.org/ downloads>. Acesso: 3 ago. 2015.

ELTON, C.; NICHOLSON, M. The ten-year cycle in numbers of the lynx in Canada. Journal of Animal Ecology, v.11, n.2, p. 215-244, 1942.

GAUSE, G. F. The struggle for existence. Baltimore: Willians \& Wilkins Company. 1934. 163p.

GILPIN, M.E. Spiral chaos in a predador-prey model. The American Naturalist, v.113, p.306-308, 1979.

GOTELLI, N.J. Ecologia. ed. 3. Planta: Londrina, Brasil. 2007. 260p.

HALL, C. A. S. An assessment of several of the historically most influential theoretical models used in ecology and of the data provided in theory support. Ecological Modelling, v.43, p.4-31, 1988.

HIGLEY, L. G. 2002. Lecture 12: Populations and Biotic Potential. Disponível em: <http://entomology. unl.edu/lgh/ent806/Lecture12_populations.htm. 2002>. Acesso: 22 ago. 2015.

HOUAISS, A. Dicionário Houaiss da língua portuguesa. Rio de Janeiro: Objetiva. 2009.

LEGENDRE, P.; LEGENDRE, L. Numerical Ecology. 2 ed. Elsevier: Amsterdam. 1998. 853 p. 
LOTKA, A.J. Elements of Physical Biology. Williams and Wilkins. Baltimore. 1925. 460 pp.

MALTHUS, T. R. 1798. An Essay on the principle of population. 1 ed. London: J. Johnson. Disponível em: $<$ http://www.esp.org/books/malthus/population/malthus.pdf >. Acesso: 17 ago. 2015.

MANSSON, B. A.; MCGLADE, J. M. Ecology, thermodynamics and H.T. Odum's conjectures. Oecologia, 93: 582-596, 1993.

MARGALEF, R. Ecologia. Omega: Barcelona. 1982

MOLTER, A; RAFIKOV, M. Controle ótimo para um sistema caótico de Lotka-Volterra. TEMA - Sociedade Brasileira de Matemática Aplicada e Computacional, v.5, n.2, p.239-248, 2004.

ODUM, E.P. Ecologia. Guanabara Koogan: Rio de Janeiro, Brasil. 1988. 434p.

PINTO-COELHO, R. M. Fundamentos em ecologia. Artmed: Porto Alegre, Brasil. 2000. 247p.

RICKLEFS, R. E. A Economia da natureza. ed. 6. Guanbara Koogan: Rio de janeiro, Brasil. 2010. 503p.

SCHRUM, J. 2005. Modeling Population Dynamics withVolterra-Lotka Equations in Partial Fullfillment of the Mathematics Capstone. Disponível em: <http://www.cs.utexas.edu/users/schrum2/MathCapstone. pdf $>$. Acesso: 20 ago. 2015.

SCOTT, P. A.; CRAINE, I. T. M. The Lynx cycle: a climatic perspective. Climate Research, v.2, p.235-240, 1993.

SHIH, S. D. The period of a Lotka-Volterra system. Taiwan Journal of Mathematics. v.1, n.4, p.451-470, 1997.

SOUTHWOOD, T. R.E. Ecological methods with particular reference to the study of insect populations. Champman and Hall: London. 1971.

SZMRECSÁNYI, T. Thomas Robert Malthus: economia. São Paulo: Ática, 1982.

VANCE, R. R. Predation and resource partitioning in one predator - two prey, model community. The American Naturalist, v.112, p.797-813, 1998.

VENSIN. 2015. Vensin from Ventana System Inc. versão 6.0. Disponível em: <http://www.vensim.com». Acesso: 3 ago. 2015.

VERHULST,P. F. Notice sur la loi que la population suit dans son accroissement. Corresp Math Phys, v.10, p.113-121, 1838.

VERHULST, P. F. Recherches mathématiques sur la loi d'accroissement de la population. Nouv Mém Acad Royale Sci Bell Let Brux, v.18, p.1-41, 1845.

VERHULST, P.F. Deuxième mémoire sur la loi d'accroissement de la population. Mém Acad Royale Sci Let Beaux Arts Belg, v.20, p.1-32, 1847.

VOLTERRA, V. "Variazioni e fluttuazioni del numero d'individui in specie animali conviventi". Mem. Acad. Lincei Roma, v.2, p.31-113, 1926.

VOLTERRA, V. Variations and fluctuations of the number of individuals in animal species living together. Animal Ecology, Chapman, RN (ed). New York: McGraw-Hill, 1931. 